\title{
MICROBIOLOGY
}

\section{Bacteria make the rotor go round}

The gliding bacteria Mycoplasma mobile yoked to an inorganic rotor will turn it along a circular track, generating a tiny biological hybrid motor.

We humans have been putting lesser organisms to work for us since the dawn of civilization. But whereas a workhorse might have powered a grist mill in the olden days, scientists are now harnessing a bacterium to form a tiny hybrid micromachine. It is an exploitation that probably will not bother even the most stalwart animal-rights activist.

Yuichi Hiratsuka, of the Japan Advanced Institute of Science and Technology, and his colleagues at the National Institute of Advanced Industrial Science and Technology in Japan have for some time now been interested in making tiny hybrid devices combining biological elements, such as motor proteins, with inorganic materials. Recently they were inspired by a presentation by Makoto Miyata from Osaka City University, now a close collaborator, who

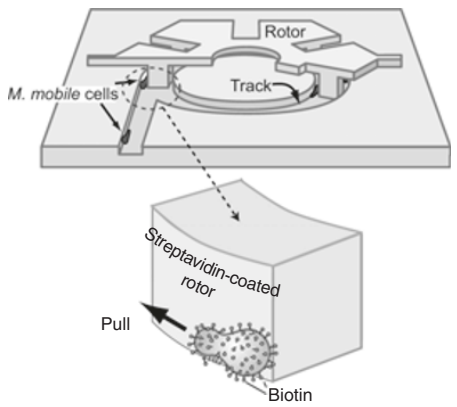

Figure 1 | Biotinylated M. mobile bacteria turn a streptavidin-coated rotor along a circular track. Copyright 2006, National Academy of Sciences, USA.

showed a movie of M. mobile gliding unidirectionally on a glass surface. "It immediately occurred to us that we should be able to make microrotary motors using these bacterial cells," says Hiratsuka.

Using photolithography, they created a sunken, circular track coated with sialic proteins to restrict the movement of the bacteria. To yoke the bugs to a rotor designed to move along the track, they covered the surface of the rotor with streptavidin molecules and chemically biotinylated cell-surface proteins on M. mobile (Fig. 1). They captured movies of the bugs turning the rotor with a rotation rate of $\sim 1.5-2.6$ r.p.m.

Hiratsuka and colleagues hope to put their hybrid motor to work, for example, as a micropump in lab-on-a-chip systems. In the future, "we would like to make microrobots driven by biological motors, which would move around and do mechanical work in a micrometer world," says Hiratsuka. Though this is the first description of a bacteriadriven microdevice, he believes that various micro-organisms with interesting motility properties could be exploited to create other exciting hybrid devices.

\section{Allison Doerr}

\section{RESEARCH PAPERS}

Hiratsuka, Y. et al. A microrotary motor powered by bacteria. Proc. Natl. Acad. Sci. USA 103, 13618-13623 (2006).

\section{PROTEIN BIOCHEMISTRY}

\section{SWEETENING UP YEAST GLYCOPROTEIN SYNTHESIS}

An engineered yeast strain is capable of carrying out the full range of human protein glycosylation reactions.

Making proteins in mammalian expression systems is not always ideal, but sometimes it is necessary if you want your protein to contain human posttranslational modifications. The more userfriendly bacterial and yeast systems are not naturally equipped to carry out the multitudes of unique modification reactions. Mammalian expression systems, however, suffer in two major areas; one, that it takes much more time to get sufficient amounts of protein, and two, that it is difficult to uniformly control the posttranslational modifications to make a heterogeneous product.

Luckily, Tillman Gerngross, and his colleagues at Dartmouth College and GlycoFi Inc., did not take this state of affairs for granted. After many years of work, they have succeeded in engineering an artificial Pichia pastoris yeast strain that is capable of performing the entire range of human glycosylation reactions, formerly limited to mammalian culture systems. They achieved this by knocking out four genes coding for yeast glycosylation enzymes and introducing 14 heterologous genes encoding human glycosylation enzymes. This feat was not nearly as simple as it might sound, and as Gerngross explains, "you can't just take a human enzyme and pull it into the yeast because the targeting mechanism will be scrambled...you have to target specific enzymes to the secretory pathway of the yeast in a way where the sequential nature of the reactions is maintained."
Gerngross and his colleagues are particularly interested in making glycosylated human proteins for therapeutic purposes, so they used their engineered yeast strain to synthesize the heavily glycosylated erythropoietin, an important therapeutic protein for the treatment of anemia. Erythropoietin made in the humanized strain was functional in mice, whereas erythropoietin made in wild-type $P$. pastoris cells was nonfunctional. "In the past, we've relied on ways of making [therapeutic proteins] that did not afford us the opportunity to control their composition, in particular as it relates to glycosylation," says Gerngross, stressing that "If you can't control your final composition and you're making a drug that goes into people, that's not a good thing."

This work could also have a substantial impact on basic research; as Gerngross explains, "with this tool, you can make a protein in its various glycoforms and test them individually for their function.... The community has known for many years that glycosylation impacts activity, but there was no good way of really figuring it out." With the aid of such cleverly engineered yeast strains, finicky mammalian expression systems may in the future become obsolete.

\section{Allison Doerr}

RESEARCH PAPERS

Hamilton, S.R. et al. Humanization of yeast to produce complex terminally sialylated glycoproteins. Science 313, 1441-1443 (2006). 